\title{
DIGITALCOMMONS
}

@WAYNESTATE-

Wayne State University

\section{Associations between Maternal Psychosocial Stress, DNA Methylation, and Newborn Birthweight Identified by Investigating Methylation at Individual, Regional, and Genome Levels}

\author{
Christopher J. Clukay \\ University of Florida \\ David A. Hughes \\ University of Bristol \\ Darlene A. Kertes \\ University of Florida \\ Connie J. Mulligan \\ University of Florida
}

Follow this and additional works at: https://digitalcommons.wayne.edu/humbiol_preprints

\section{Recommended Citation}

Clukay, Christopher J.; Hughes, David A.; Kertes, Darlene A.; and Mulligan, Connie J., "Associations between Maternal Psychosocial Stress, DNA Methylation, and Newborn Birthweight Identified by Investigating Methylation at Individual, Regional, and Genome Levels" (2020). Human Biology Open Access Pre-Prints. 164.

https://digitalcommons.wayne.edu/humbiol_preprints/164

This Article is brought to you for free and open access by the WSU Press at DigitalCommons@WayneState. It has been accepted for inclusion in Human Biology Open Access Pre-Prints by an authorized administrator of DigitalCommons@WayneState. 
Associations between Maternal Psychosocial Stress, DNA Methylation, and Newborn

Birthweight Identified by Investigating Methylation at Individual, Regional, and Genome

Levels

Christopher J. Clukay,1,2* David A. Hughes,3,4 Darlene A. Kertes,2,5 and Connie J. Mulligan1

1Department of Anthropology, University of Florida, Gainesville, Florida, USA.

${ }_{2}$ Genetics Institute, University of Florida, Gainesville, Florida, USA.

3MRC Integrative Epidemiology Unit, University of Bristol, Bristol, BS8 2BN, UK.

3Population Health Sciences, Bristol Medical School, University of Bristol, Bristol, BS8 2BN, UK.

5Department of Psychology, University of Florida, Gainesville, Florida, USA.

*Correspondence to: Christopher J. Clukay, 2033 Mowry Rd., PO Box 103610, University of

Florida, Gainesville, FL 32608 USA. E-mail: cjclukay@ufl.edu.

Short Title: Maternal Stress and Methylation across Genomic Levels

KEY WORDS: WAR TRAUMA, DEMOCRATIC REPUBLIC OF CONGO, EPIGENETIC

CLOCK, VARIABLY METHYLATED REGION, EWAS, STRESS EPIGENETICS, SEXUAL VIOLENCE

Pre-print version. Visit http://digitalcommons.wayne.edu/humbiol/ after publication to acquire the final version. 


\section{Abstract}

Stress is known to affect health throughout life and into future generations, but the underlying molecular mechanisms are unknown. We tested the hypothesis that maternal psychosocial stress influences DNA methylation (DNAm), which in turn impacts newborn health outcomes. Specifically, we analyzed DNAm at individual, regional, and genome-wide levels in order to test for associations with maternal stress and newborn birthweight. Maternal venous blood and newborn cord blood were assayed for methylation at $\sim 450,000 \mathrm{CpG}$ sites $(\mathrm{n}=24$ and 22 , respectively). Methylation was analyzed by examining $\mathrm{CpG}$ sites individually (epigenome-wideassociation-study [EWAS]), as regional groups (variably methylated region [VMR] analysis in maternal blood only), and through epigenome-wide measures (genome-wide mean methylation [GMM], Horvath's epigenetic clock, and mitotic age). These methylation measures were tested for association with three measures of maternal stress (maternal war trauma, chronic stress, and experience of sexual violence) and one health outcome (newborn birthweight). We observed that maternal experiences of war trauma, chronic stress, and sexual assault were each associated with decreased newborn birthweight ( $\mathrm{p}$-value $<1.95 \times 10-7$ in all cases). Testing individual CpG sites using EWAS, we observed no associations between DNAm and any measure of maternal stress or newborn birthweight in either maternal or cord blood after Bonferroni multiple testing correction, although the top ranked $\mathrm{CpG}$ site in maternal blood that associated with maternal chronic stress and sexual violence before multiple testing correction is located near the SPON1 gene. Testing at a regional level, we found increased methylation of a VMR in maternal blood near SPON1 that was associated with chronic stress and sexual violence after Bonferroni multiple testing correction ( $\mathrm{p}$-value $=1.95 \times 10-7$ and $8.3 \times 10-6$, respectively). At the epigenomic level, cord blood GMM was associated with significantly higher levels of war trauma (p-value 
0.025 ) and suggestively associated with sexual violence $(\mathrm{p}$-value $=0.053$ ). The other two epigenome-wide measures were not associated with maternal stress or newborn birthweight in either tissue type. Despite our small sample size, we identified associations even after conservative multiple testing correction. Specifically, we found associations between DNAm and the three measures of maternal stress across both tissues, specifically a VMR in maternal blood and GMM in cord blood were both associated with different measures of maternal stress. The association of cord blood GMM, but not maternal blood GMM, with maternal stress may suggest different responses to stress in mother and newborn. It is noteworthy that we only found associations when $\mathrm{CpG}$ sites were analyzed in aggregate, either as variably methylation regions (VMRs) or a broad summary measure of genome-wide mean methylation (GMM).

Pre-print version. Visit http://digitalcommons.wayne.edu/humbiol/ after publication to acquire the final version. 
Psychosocial stress, the physical and psychological stress resulting from social forces, has a well-established relationship to human health (Lehrner and Yehuda, 2018; D’Andrea et al., 2011; Massart et al., 2014; Jawaid et al., 2018). Psychosocial stress can result from either chronic stressors, such as daily experiences of unfair treatment (Quinlan et al., 2016), or acute stressors that leave lasting psychological effects, such as exposure to conflict related violence (Dajani et al., 2018). Understanding how these stress exposures impact biology, and the mechanisms by which this occurs, are key to fully assessing the impact of psychosocial stress.

The molecular mechanisms by which stress affects biology remain to be fully elucidated. In recent years, DNA methylation (DNAm) has garnered increasing attention as a possible mechanism to translate stressful experiences into altered health outcomes. DNA methylation is a process by which a methyl group is added to a cytosine nucleotide, most commonly one located next to a guanine (called a $\mathrm{CpG}$ site). Methylated $\mathrm{CpGs}$ are referred to as epigenetic marks whose presence or absence act as genomic regulators that influence cellular biology, development, and disease (Smith and Meissner, 2013; Cedar and Bergman, 2009; Lister et al., 2009; Roadmap Epigenomics Consortium et al., 2015). Studies have linked DNAm changes to numerous psychosocial stressors including socio-economic status, depression, childhood maltreatment, and learned fear experiences tied to PTSD (Braithwaite et al., 2015; Appleton et al., 2013; Cao-Lei et al., 2014; Zovkic and Sweatt, 2013; Barfield et al., 2014). There is also evidence that such changes in DNAm may be transmitted to future generations, such as a mother's stressful experience resulting in offspring exposure in utero (Braithwaite et al., 2015; Cao-Lei et al., 2014; Mulligan et al., 2012; Heijmans et al., 2008; Tobi et al., 2014).

A primary function of DNAm is to facilitate and maintain cell type differentiation (Houseman et al., 2012), or aid in producing different cell types among populations of cells with 
the same genomic sequence. This process of cell differentiation (controlled primarily by gene regulation and partly by DNAm) begins early in embryogenesis when parental DNAm patterns are erased and new ones are established via a set of proteins known as the methylation complex (Hata et al., 2002; Fatemi et al., 2002). Many of these same proteins then act to maintain, and possibly alter, DNAm patterns throughout development and into adulthood. Changes in DNAm are often associated with changes in gene expression, or the amount of messenger RNA resulting from a gene. The nature of this effect is complex and, while promoter methylation is often associated with decreased expression, the effect of DNAm at other genomic locations, such as in the gene body or at enhancers, can be associated with either increased or decreased gene expression depending on the specifics of the region (Wagner et al., 2014).

Thus, any changes in DNAm due to psychosocial stress will occur in the context of these larger cell type effects and the challenge is to detect such changes above and beyond this natural variation. As such, it is generally accepted that the impact of psychosocial stress on DNAm will be small and possibly restricted to specific loci or parts of the genome (Mulligan, 2016). It also means that differences in how we conceptualize and analyze DNAm may influence our results. When considering the factors above, there are at least three general possibilities regarding how psychosocial stress may impact DNAm and how DNAm may impact gene expression, resulting in altered health outcomes:

- Site-specific methylation changes are made at individual CpG sites.

- Specific genomic regions are affected, meaning groups of CpG sites are altered together in response to a stressor.

- A generalized response to stress results in changes across the epigenome, altering $\mathrm{CpG}$ sites at multiple, disparate locations. 
These concepts are not mutually exclusive; combinations are possible. They also stand in contrast to candidate gene approaches, which we have performed previously and will be discussed later. Unlike candidate gene approaches that are teleological in nature, due to their selection being based on gene product function, the concepts investigated in the current study represent distinct mechanistic possibilities for DNA methylation itself being affected and are unbiased with respect to gene function. Each concept has specific classes of analytical methods associated with them.

The investigation of individual $\mathrm{CpG}$ sites is commonly explored using an epigenomewide association study (EWAS). In an EWAS, each site is tested individually for association with the variables of interest. An EWAS is the most common form of large-scale epigenetic analysis.

Genomic regions can be explored via multiple methods depending on the nature of the data available (Clukay et al., 2018; Jaffe et al., 2012). One particularly powerful method is the variably methylated region (VMR) technique outlined by Garg et al. (2018). Based on the concept that genomic regions exhibiting higher inter-individual variability are more likely to show an association with environment, VMRs are defined as sections of the genome with at least three $\mathrm{CpG}$ sites in the upper 5 th percentile of variability within the sample, as measured by standard deviation. This technique allows for a more focused approach based upon theory and data, thus increasing power.

Finally, the third concept is often implicitly applied when using epigenome-wide measures of DNAm. These can include simple calculations, such as genome-wide mean methylation (GMM), or more complex measures, such as the epigenetic clock developed by Horvath (2013) to measure biological aging or the mitotic age measure developed by Youn and 
Wang (2018) to measure number of cell divisions. Changes in broad measures such as GMM, which measures the average methylation level of all autosomal CpG sites ( 400,000 sites on the Illumina Human-Methylation 450 Bead Chip, for example), capture epigenetic variation across the genome in one measure and can be seen as potential indicators of cellular stress or dysregulation (Carnell and Goodman, 2003). Meanwhile, the epigenetic clock, a weighted average of approximately $400 \mathrm{CpG}$ sites throughout the genome associated with age, is an estimate of methylation age calibrated to chronological human aging in those tissues. Initial iterations of the clock were purposefully related to some changes in cell type as cell composition can change with age (Horvath, 2013), but later versions were rendered independent of cell type (Horvath et al., 2016; Horvath et al., 2018). Individuals exhibiting increased epigenetic age compared to their chronological age, known as epigenetic age acceleration, may have an increased risk for age-related diseases (Horvath et al., 2016). In contrast, mitotic age estimates reflect cellular turnover or the average number of cell divisions a population of cells has undergone rather than an individuals' chronological age. Different tissues from the same individual can have significantly different mitotic ages depending on how quickly cells divide within that tissue. While mitotic age is a recently developed measure, epigenetic age has been previously observed to associate with stress in multiple studies (Zannas et al., 2015; Verhoeven et al., 2018; Fiorito et al., 2017). However, given that the precise manner in which DNAm acts in a cell is unknown, both measures may be of interest when studying psychosocial stress.

Here, we continue an ongoing study of the effects of psychosocial stress on mother-infant dyads from the Democratic Republic of the Congo. Previously, we tested candidate genes and reported associations between maternal stress and DNAm in multiple genes in mother-infant dyads (Kertes et al., 2017; Montoya-Williams et al., 2017; Rodney and Mulligan, 2014; Kertes et 
al., 2016). Specifically, we reported associations connecting both maternal stress and birthweight to methylation at $N R 3 C 1$, a gene involved in the stress response, in newborn cord blood (Mulligan et al., 2012; Kertes et al., 2016). We also found associations between DNAm in the methylation complex genes and GMM in mothers, suggesting possible avenues for genome-wide changes in methylation as a result of stress (Clukay et al., 2018).

Collectively, our previous results suggest that DNAm patterns have changed in response to maternal stress and that associations between DNAm and birthweight also exist. However, the relationship between stress-associated variation in DNAm and birthweight remains unclear. Here, we continue to use this mother-infant dyad framework to test for associations between maternal stress and methylation variation in mothers and their newborns. The three methodologies (EWAS, VMRs in maternal blood, and epigenome-wide summary measures) outlined above will be used. Methylation was tested for association with two previously constructed summary variables of maternal stress, war trauma and chronic stress (Kertes et al., 2016; Kertes et al., 2017), and an additional variable related to sexual violence due to previous findings indicating the salience of the stressor (Mulligan, 2016). Lastly, these measures of methylation were also compared to birthweight as a health outcome.

\section{Materials and Methods}

\section{Sample and Data Collection}

Sample collection and descriptions of sociocultural data for this project are discussed in detail in Rodney and Mulligan (2014). In brief, this pilot phase of the project recruited 25 mother-infant dyads from HEAL Africa hospital in Goma, DRC. Recruitment was conducted in the summer of 2010 with mothers being interviewed and blood samples taken shortly after delivery. All women 
who gave birth during that time period were eligible for inclusion in the study. The study was approved by the Western Institutional Review Board, Olympia, WA (www.wirb.com, WIRB Project \#20100993).

Anthropometric measurements, including birthweight, were collected at the time of birth. Venous blood was collected from mothers shortly after birth and cord blood was collected immediately after birth. DNA was extracted using QIAamp DNA Mini Kits (Qiagen, Cat No. 51304) per manufacturer specifications. Sociocultural data concerning maternal stress exposures were collected using semi-structured interviews and standardized surveys based on Brunet et al. (2001). The beginning of the interview was conversational in nature to build rapport, followed by administration of the survey. Survey instruments were translated into the Congolese dialect of Swahili and modified for use in the DRC to ensure they addressed cultural stressors relevant to the population. A list of questions asked, translated into English, is available on Mendeley Data (DOI: $10.17632 / \mathrm{wwgw} 2 \mathrm{ct} 2 \mathrm{wv} .1)$.

\section{Measures of Maternal Stress}

Summary measures of stress were constructed as described in Kertes et al. (2016). In sum, factor analysis was used to identify two major factors explaining $55 \%$ of the variance. All but two questions within those factors contributed to internal consistency, the exceptions being in-law stress and a question regarding co-wives, both of which were removed. After processing for internal consistency using Cronbach's $\alpha(\alpha>0.8$ for each measure after removal of inconsistent questions), the two factors were identified as representing chronic stress and war trauma.

In addition to the two summary stress measures, a third measure of severe sexual violence, reflecting personal experience of rape, was also tested. Sexual violence was commonly 
used as a weapon of war in the Democratic Republic of the Congo and we have previously reported associations between this measure and birthweight (Mulligan, 2016). Multiple questions were asked regarding whether the mother had experienced rape in the past, during the pregnancy, conceived a child previously due to rape, or whether the current pregnancy was due to rape. Each 'yes' answer increased the score by one, resulting in a maximum possible score of four and a minimum possible score of zero.

All sample characteristics, stress measures, and covariates are available on Mendeley Data (DOI: 10.17632/wwgw2ct2wv.1)

\section{Generation and Processing of DNA Methylation Data}

DNA methylation was assayed using the Illumina Human-Methylation 450 Bead Chip (Illumina, USA) per the manufacturer's specifications. The unprocessed methylation data are available on the Gene Expression Omnibus (GSE54399). Due to space constraints on the chip, only 24 of the dyads were assayed. All internal control and quality probes were run on the chip as part of the standard processing using the meffil packages, including background correction and removal of batch effects. Maternal and cord blood samples were randomized across the chip. Additionally, two cord samples were later found to be contaminated with adult venous blood and were removed from the analysis.

All data processing was performed using $\mathrm{R}$ unless otherwise noted ( $\mathrm{R}$ Development Core Team, 2015). While original processing for Rodney and Mulligan (2014) was conducted using the minfi package developed by Aryee et al. (2014), advancements in processing tools, batch correction, and normalization prompted the reprocessing of the data for the current study using the meffil package (Min et al., 2018). Coding and specific parameters used were based on those 
recommended by the package. Both the code used and the plate layout necessary for running the code are available on Mendeley Data (DOI: 10.17632/wwgw2ct2wv.1). Beta values were normalized using functional normalization via the meffil package. Following normalization, $\mathrm{CpG}$ sites were then masked (i.e. removed) based on recommendations for the African superpopulation by Zhou et al. (2016), resulting in a final total of 412,117 CpG sites.

After processing, cell type proportions for maternal blood and cord blood were estimated separately using the meffil package with reference datasets based on Houseman et al. (2012) and de Goede et al. (2015), respectively. Principal component analysis (PCA) was performed on cell type proportions and PCs accounting for $95 \%$ or more of the variance were included as covariates in all subsequent analyses to account for cell type heterogeneity. This resulted in four cell type PCs being included as covariates in relation to maternal blood and five when testing cord blood, due to the increased number of cell types in cord blood (nucleated red blood cells, for example). Surrogate variables for batch were identified using the meffil.ewas() function, two in maternal blood and three in cord blood, using the option sva $=\mathrm{T}$, in meffil. These surrogate variables were also included as covariates for all analyses related to methylation. Additional covariates for each tissue type are defined below.

\section{Analyses of DNA Methylation}

All analyses were conducted in R unless otherwise noted (R Development Core Team, 2015). Associations in maternal blood and cord blood were tested separately for all analyses. Tests of association with maternal blood methylation were corrected for maternal age and tests of association with cord blood methylation were corrected for infant sex. All tests of association with birthweight, regardless of tissue, were corrected for infant sex. 
Epigenome-wide association study. EWAS analyses were conducted using the meffil.ewas() function native to meffil. For EWAS analyses, the multiple testing threshold was calculated using Bonferroni correction based on the number of individual sites tested [0.05/(412,360 sites) $=1.21 \times 10-7]$.

VMR identification. VMRs in maternal blood were identified using the requirements and scripts described by Garg et al. (2018). CpG sites were marked as part of a VMR using a sliding, 1,000basepair window. Two criteria were required for the $\mathrm{CpG}$ sites within a window to be marked as part of a VMR. First, at least three $\mathrm{CpG}$ sites within the window were required to be in the top $5 \%$ of variability within our sample, as measured by standard deviation. Second, at least half of the total sites within the window needed to fulfil these criteria in order to ensure that the majority of the sites within the region actually varied within the sample. The $\mathrm{CpG}$ site exhibiting the highest variance within each VMR was then chosen as a representative site and tested for association with the variables of interest using the meffil.ewas() function in meffil. Since separation by sex was necessary for VMR identification in order to prevent confounding, there was insufficient variation among cord blood samples to identify or test VMRs for association.

Epigenome-wide measures of DNA methylation. Three summary measures of methylation were calculated in this study; GMM, Horvath's epigenetic clock (Horvath et al., 2018), and mitotic age (Youn and Wang, 2018). GMM was calculated as an individual's average level of methylation for all autosomal CpG sites after data processing and filtering. Since GMM is an average across all sites, even small changes in GMM may reflect large shifts in gene regulation. 
Horvath's epigenetic clock for skin and blood cells, known as the skin and blood clock, reflects changes in the epigenome associated with chronological aging in those tissue. This measure was developed specifically for use in skin and blood cells to compensate for inaccuracies of the pantissue clock due to fibroblasts (Horvath et al., 2018). Furthermore, the skin and blood clock has been specifically shown to exhibit higher accuracy and less error when measuring cord blood samples, which our study examines. The measure was calculated for each individual using filtered methylation values and the R code provided by Horvath et al. (2018). Mitotic age was calculated using filtered methylation values and the R code provided by Youn and Wang (2018). The measure uses a probability model to calculate the average number of cell divisions based on reference data. It is worth noting that there is an offset in the measure, meaning that mitotic age values should not be taken as the absolute number of cell divisions. However, the offset is constant across samples, meaning the samples remain internally comparable.

\section{Results}

\section{Sample Characteristics}

The average age of study participants was 26.9 years old and average birthweight was 7.00 lbs. (Table 1). Stress was estimated using three summary statistics. First, war trauma, a measure of war-related stressors including exposure to violent conflict, exposure to sexual violence, and refugee status, had an average of 1.58 with a range from 0 to 8 . Second, chronic stress, a measure of daily stressors including questions such as access to adequate food, difficulty paying bills, and being ashamed to cry, had an average of 6.54 with a range from 0 to 18 . Finally, $29 \%$ or seven mothers had suffered a personal experience of rape with scores on the sexual violence measure ranging from 0 to 3 . This prevalence is comparable to that observed in the United States, where it 
is estimated that approximately one in five women will experience rape in their lifetime (Smith et al., 2018).

\section{Associations between Maternal Stress and Newborn Birthweight}

The effect of maternal stress on newborn health outcomes is well known (Lehrner and Yehuda, 2018; D’Andrea et al., 2011; Massart et al., 2014; Jawaid et al., 2018). We have previously reported an association between maternal psychosocial stress and newborn birthweight in this sample using slightly different measures of maternal stress (Mulligan et al., 2012). Here we tested three measures of maternal stress (war trauma, chronic stress, and sexual violence) for association with newborn birthweight. All three measures negatively correlated with birthweight (Table 2) and explained between $35 \%$ and $46 \%$ of the total variation in birthweight. Each unit of the war stress and chronic stress scales was associated with $0.49 \mathrm{lbs}$. (p-value $=9.0 \times 10-4)$ and 0.18 lbs. (p-value $=3.0 \times 10-4)$ lower birthweight per unit of stress, respectively. Each point of the sexual violence scale was associated with a $0.91 \mathrm{lbs}$. lower birthweight ( $\mathrm{p}$-value $=2.3 \times 10-3$ ). These associations constitute the foundation we use to test DNAm as one possible mechanism by which maternal stress impacts health outcomes.

\section{Analysis of DNA Methylation Associations with Maternal Stress and Birthweight}

In order to test for an effect of maternal stress on DNAm, and an effect of DNAm on newborn birthweight, we tested the DNAm data using three different methodologies: (1) EWAS to test individual CpG sites, (2) VMR analyses to test methylation in specific regions, and (3) methylation summary measures to test epigenome-wide changes in methylation. 


\section{EWAS as a Measure of Site-Specific DNA Methylation}

EWAS was used to test each CpG site individually for association with maternal stress and newborn birthweight. After multiple testing correction (Bonferroni Threshold = 1.21x10-7), no $\mathrm{CpG}$ sites in maternal or cord blood were found to be individually associated with maternal war trauma, chronic stress, or sexual violence (Figure 1A-F). In addition, no significant associations were identified between birthweight and individual $\mathrm{CpG}$ sites in either maternal venous blood or newborn cord blood (Figure 1G-H). The CpG site with an association closest to our confidence threshold $(\mathrm{cg} 23284931 ; \mathrm{p}$-value $=1.95 \times 10-7)$ was observed in maternal blood with maternal chronic stress as the exposure (Figure 1C). The gene associated with this region, SPON1, has been previously associated with changes to both DNA methylation and gene expression in association with PTSD (Mehta et al., 2013), as well as with Vitamin D levels, Alzheimer's disease, brain connectivity, and numerous blood protein levels (Manousaki et al., 2017; Sherva et al., 2014; Jahanshad et al., 2013; Sun et al., 2018). This CpG site is also the top ranked site in maternal blood with maternal experience of sexual violence as the exposure ( $\mathrm{p}$-value $=8.3 \times 10-6)$.

\section{VMR Analysis as a Measure of Regional DNA Methylation}

VMR analysis identifies highly variable regions of methylation in which multiple $\mathrm{CpG}$ sites are changing within the particular set of samples being tested. This variability increases the likelihood of a region being sensitive to environmental stressors. Since VMR analysis requires separation by sex for VMR identification, there was only sufficient sample size to accurately determine variance in the maternal blood samples.

VMR analysis identified a total of 885 regions in maternal blood (see supplemental material for a list of all VMRs and constituent sites). Of these VMRs, 549 contained CpG sites 
previously identified in whole blood VMRs by Garg et al. (2018). Using the CpG site with the highest variability for each VMR as a representative, each VMR was then tested for association with maternal stress and newborn birthweight.

After multiple test correction for the number of VMRs identified (Bonferroni Threshold= $0.05 / 885=5.65 \times 10-5)$, two associations were observed in maternal venous blood with the same CpG site, cg23284931. The VMR represented by CpG site cg23284931 associated with maternal chronic stress with an effect estimate of 0.025 per unit of stress ( $\mathrm{p}$-value $=1.95 \times 10-7$; Figure $2 \mathrm{~A}$ ) and associated with experience of sexual violence with an effect estimate of 0.15 per point of the sexual violence measure ( $\mathrm{p}$-value $=8.3 \times 10-6$; Figure $2 \mathrm{~B})$. The VMR itself occurs near the SPON1 gene and consists of ten $\mathrm{CpG}$ sites, all of which are positively correlated with one another (correlation coefficient $>0.45$ in all cases). The representative $\mathrm{CpG}$ site also exhibited the strongest potential association between the same variables in the EWAS analyses described above, i.e. methylation at cg23284931 in maternal blood associated with both chronic stress and experience of sexual violence before multiple testing correction. In the VMR analysis, no tests using birthweight or maternal war trauma met the Bonferroni confidence threshold for significance.

\section{GMM and Methylation Ages as Epigenome-Wide Measures of DNA Methylation}

Three epigenome-wide measures of DNAm were tested; GMM, the epigenetic clock for skin and blood cells (Horvath et al., 2018), and the mitotic age measure of cell replications (Youn and Wang, 2018). These three epigenome-wide measures were generated for both maternal and cord blood and tested separately for association with maternal stress measures (war trauma, chronic stress, and sexual violence) as well as newborn birthweight (Table 3). 
Maternal war trauma was associated with cord GMM with an effect estimate of $-4.8 \times 10-4$ per unit of stress $(p$-value $=0.025 ;$ Figure $3 \mathrm{~A})$. One suggestive association $(\mathrm{p}$-value between 0.05 and 0.06) was observed between cord GMM and maternal experience of sexual violence with an effect estimate of $-7.8 \times 10-4$ per unit stress ( $\mathrm{p}$-value $=0.053$, Figure $3 \mathrm{~B})$. Thus, decreased cord GMM was found to be associated with higher levels of maternal war trauma and experience of sexual violence. These associations were not seen with maternal GMM.

No associations were observed between maternal stressors or birthweight with epigenetic age or mitotic age in either maternal or cord blood methylation (Table 3). It is noteworthy that correction for chronological age in maternal blood when calculating epigenetic age acceleration can be accomplished in two ways; simple subtraction of chronological age from epigenetic age or by including chronological age as a regression variable. Using the subtraction method, we found that epigenetic age was significantly higher than chronological age (Supplemental Figure S1A; mean increase $=4.2$ yrs., p-value $=7.1 \times 10-6$ ). However, the inclusion of maternal age as a regression variable, even as the sole term in the model, followed by taking the residuals to determine maternal age acceleration, completely removed this age acceleration effect (Supplemental Figure S1B; mean increase $=-0.093$ yrs., p-value $=0.89)$. These conflicting results suggest that the increase in epigenetic age seen when subtracting chronological age is an artifact of the epigenetic age calculation and is not a valid increase in epigenetic age, an artifact which has been noted in previous studies (Dhingra et al., 2019).

\section{Discussion}

This study expands upon our previous work investigating the effects of maternal stress on DNAm and newborn birthweight. We have previously observed associations between maternal 
stress, DNAm, and birthweight using candidate gene approaches (Kertes et al., 2017; MontoyaWilliams et al., 2017; Rodney and Mulligan, 2014; Kertes et al., 2016). We hypothesize that the mechanism by which DNAm is altered may result in different patterns of association among CpG sites, leading us to test for associations between maternal stress, newborn birthweight, and DNAm changes at individual, regional, and epigenome-wide levels. In the current study, significant associations were found when analyzing DNAm sites in aggregate at regional and epigenome-wide levels.

Using VMR analysis to test for regional associations, one VMR region, near the SPON1 locus, was associated with chronic stress and sexual violence in maternal venous blood (Figure 2). In a study by Mehta et al. (2013), the representative CpG site at this VMR locus (cg23284931) and other sites in the region were found to associate with childhood maltreatment. Specifically, they found that individuals with PTSD exhibited higher levels of average methylation in the region compared to controls, similar in direction to our study, which found increased VMR methylation in association with stress and sexual violence. Using microarrays to measure gene expression, Mehta et al. (2013) then verified that the changes in methylation were also associated with expression changes in SPON1. While individuals in our sample were not surveyed for PTSD symptoms, our findings are consistent with the premise that specific genomic regions may be targeted for methylation change.

Indeed, other studies support the conclusion that regional methylation patterns are associated with psychosocial stress as well. Previous studies have found altered regions of DNAm in relation to stressors, such as childhood abuse (Shields et al., 2016; Suderman et al., 2014), and mental disorders such as major depressive disorder (Nieratschker et al., 2014). As 
such, more complex methods of examining regional changes to methylation, such as VMR used here and the bump hunting method developed by Jaffe et al. (2012), are warranted.

Maternal war trauma appears to influence epigenome-wide DNAm, as measured by cord blood GMM, and birthweight in the same direction. Specifically, higher levels of maternal war trauma were linked with both lower cord blood GMM and lower birthweight. One could speculate that the generalized, epigenome-wide response to maternal stress could be evidence of either dysregulation or multitarget changes in gene expression in preparation for a stressful postnatal environment, as other studies have suggested (Cao-Lei et al., 2014; Suderman et al., 2014). Altered methylation levels could then lead to changes in birthweight or, alternatively, the observed differences in methylation and birthweight could both be independent effects of stress exposure. The lack of a direct association between birthweight and DNA methylation would seem to favor the interpretation that they are each independently affected by stress.

The associations with GMM reported here differ from those found in previous publications by our group, in which maternal, rather than cord blood, was observed to correlate with birthweight and stress (Rodney and Mulligan, 2014; Clukay et al., 2018). Recent improvements in functional normalization and batch correction using surrogate variable analysis prompted a reprocessing of the data using more advanced techniques (Fortin et al., 2014; Min et al., 2018). The decrease in maternal GMM in association with stress that we previously reported is similar to the decrease in cord GMM in association with stress that we report in this study. In addition, the consistency of our current VMR results with literature, including the direction of the observed effects, lends further confidence to the current analysis.

The association of cord GMM with maternal stress stands in contrast to the lack of associations observed when testing two measures of biological age, Horvath's epigenetic clock 
and mitotic age. While mitotic age is only beginning to be studied in relation to stress, our findings differ from previous studies regarding Horvath's epigenetic clock, which have reported age acceleration changes in association with PTSD in combat veterans as well as lifetime stress among African Americans (Verhoeven et al., 2018; Zannas et al., 2015). A lack of association in our study could be due to sample limitations, but it is worth noting that Verhoeven et al. (2018) and Zannas et al. (2015) used the subtraction method of correction for chronological age, which is a method that has come under scrutiny in the work of Dhingra et al. (2019) and this study. As such, our lack of significant associations between maternal stress and biological age could be an indication that the effects of stress on human health act through a different pathway than those associated with normal human aging.

The idea that stress may affect groups of $\mathrm{CpG}$ sites, as opposed to a single $\mathrm{CpG}$ site, is sensible from a biological perspective. Any effect of stress would be required to occur within the context of an active cell, meaning that not all genomic regions would be equally accessible due to chromatin structure. Factors such as packaging effects, which have been shown to interact with DNA methylation (Lee and Lee, 2012; Hashimshony et al., 2003), could affect the susceptibility of a $\mathrm{CpG}$ site to change, possibly explaining differences in signal strength between sites within a given region. For example, the manner in which DNA wraps around histones could lead some sites to be exposed for modification, while others would remain shielded or partially shielded by the protein. Furthermore, the high number of base pairs over which histones or groups of histones act would likely mean that any region that is accessible would likely not expose a single $\mathrm{CpG}$ site, but rather multiple sites.

In addition, some methyltransferase enzymes, which are primarily responsible for the establishment and maintenance of methylation patterns, are processive in nature (Svedruzic, 
2008). In this context, processivity means that these enzymes tend to methylate multiple $\mathrm{CpG}$ sites on a DNA strand before the enzyme detaches from the DNA strand. While methyltransferase enzyme levels generally decrease after birth (Kraiczy et al., 2019; Hu et al., 2008), they nonetheless provide another possible route for modification of $\mathrm{CpG}$ sites that could result in groups of sites being altered.

Furthermore, the clustering of methylated and unmethylated regions during the establishment of methylation patterns may mean that multiple $\mathrm{CpG}$ sites are associated with a given stressor even if the effect of only a select few is causative. This effect is the epigenetic equivalent of the well-established 'hitchhiker effect' seen in genetics, where variants around a causative variant are also associated with a phenotype due to selection and linkage disequilibrium (Kim and Maruki, 2011).

In addition to common pathways such as the activation of the hypothalamic-adrenalpituitary axis (Höhne et al., 2014; Eisenlohr-Moul et al., 2018), there are well documented differences between responses to acute stressors and long-term chronic stressors regarding effects such as immune function (Dhabhar, 2008). Our findings in maternal blood that the same VMR was associated with both chronic stress and sexual violence are consistent with the presence of common pathways. Still, the possibility of differing responses based on the nature of the stressor does highlight the importance of examining a variety of stressors, including extreme stressors such as the experience of sexual violence studied here.

The differences between maternal and cord blood observed when testing DNAm in relation to maternal stress (i.e. decreasing methylation in association with cord GMM and increasing methylation in association with a maternal VMR) could be due to multiple factors including differences in the effect of methylation on gene expression at those sites or differences 
in developmental stage between mothers and newborns. It is well known that increased methylation can either increase or decrease gene expression depending on the region affected (Wagner et al., 2014). As such, even though the associations with DNAm may be in opposing directions based on tissue, the downstream effect of such differences on the biological pathway may be similar.

In addition, it is worth noting that differences in response based on developmental stage are consistent with current theory. The Developmental Origins of Health and Disease hypothesis, first posited by Barker and Osmond (1986), argues for increased developmental plasticity in early development, which can lead to poor health in later life. Recent studies have begun testing changes in DNAm as a possible mechanism for this effect, well summarized in a review by Felix and Cecil (2018). Such a theory would be consistent with our observation of epigenome-wide differences in methylation associated with maternal stress in newborn cord, but not maternal venous, blood. Differing mechanisms based on developmental stage could also explain why the direction of the methylation change observed in the mothers, where increased stress was associated with increased methylation at the significant VMR, differed in direction from those observed in cord blood, where increased stress was associated with decreased GMM.

This study has several limitations worth noting. The sample size is small, which could impact our study power to differing degrees depending on the analysis. The EWAS likely has the least power, followed by the VMR analysis, relative to tests that use summary methylation measures due to the multiple testing burden. As such, it is worth repeating similar studies with larger sample sizes. However, the fact that our study found results with both VMR analysis and summary measures after conservative multiple testing correction indicates sufficient power to 
detect some associations, as well as recommending these techniques as useful tools for future studies with similar sample sizes.

Our study represents a unique examination of DNAm in relation to maternal stress through multiple analytical methods. The findings presented here provide a cautionary note that different means of conceptualizing and analyzing DNAm data can lead to different results, emphasizing the importance of considering the analytical method used in future studies of the human response to stress. Better understanding the mechanisms by which psychosocial stress may translate into epigenetic change should allow for better study design and more targeted questions from an evolutionary perspective.

\section{Acknowledgments}

This project was supported by NSF Grant \# BCS 1231264, DGE-1315138, a UF Research Opportunity Seed Fund award, and a UF College of Liberal Arts and Science award. We gratefully acknowledge the participation of the women of the Democratic Republic of the Congo and our excellent collaborators, staff and research facilities at HEAL Africa, Goma, DRC.

Received 3 December 2019; accepted for publication 4 February 2020. 


\section{Literature Cited}

Appleton, A. A., D. A. Armstrong, C. Lesseur et al. 2013. Patterning in placental 11-B hydroxysteroid dehydrogenase methylation according to prenatal socioeconomic adversity. PLoS One 8:1-7.

Aryee, M. J., A. E. Jaffe, H. Corrada-Bravo et al. 2014. Minfi: A flexible and comprehensive Bioconductor package for the analysis of Infinium DNA methylation microarrays. Bioinformatics 30:1,363-1,369.

Barfield, R. T., L. M. Almli, V. Kilaru et al. 2014. Accounting for population stratification in DNA methylation studies. Genet. Epidemiol. 38:231-241.

Barker, D. J. P., and C. Osmond. 1986. Infant mortality, childhood nutrition, and ischaemic heart disease in England and Wales. Lancet 327:1,077-1,081.

Braithwaite, E. C., M. Kundakovic, P. G. Ramchandani et al. 2015. Maternal prenatal depressive symptoms predict infant $N R 3 C 11 \mathrm{~F}$ and $B D N F$ IV DNA methylation. Epigenetics 10:408-417.

Brunet, A., D. S. Weiss, T. J. Metzler et al. 2001. The peritraumatic distress inventory: A proposed measure of PTSD criterion A2. Am. J. Psychiatry 158:1,480-1,485.

Cao-Lei, L., R. Massart, M. J. Suderman et al. 2014. DNA methylation signatures triggered by prenatal maternal stress exposure to a natural disaster: Project Ice Storm. PLoS One 9:112.

Carnell, A. N., and J. I. Goodman. 2003. The long (LINEs) and the short (SINEs) of it: Altered methylation as a precursor to toxicity. Toxicol. Sci. 75:229-235.

Cedar, H., and Y. Bergman. 2009. Linking DNA methylation and histone modification: Patterns and paradigms. Nat. Rev. Genet. 10:295-304. 
Clukay, C. J., D. A. Hughes, N. C. Rodney et al. 2018. DNA methylation of methylation complex genes in relation to stress and genome-wide methylation in mother-newborn dyads. Am. J. Phys. Anthropol. 165:173-182.

D’Andrea, W., R. Sharma, A. D. Zelechoski et al. 2011. Physical health problems after single trauma exposure: When stress takes root in the body. J. Am. Psychiatr. Nurses Assoc. $17: 378-392$.

Dajani, R., K. Hadfield, S. van Uum et al. 2018. Hair cortisol concentrations in war-affected adolescents: A prospective intervention trial. Psychoneuroendocrinology 89:138-146.

de Goede, O. M., H. R. Razzaghian, E. M. Price et al. 2015. Nucleated red blood cells impact DNA methylation and expression analyses of cord blood hematopoietic cells. Clin. Epigenetics 7:1-11.

Dhabhar, F. S. 2008. Enhancing versus suppressive effects of stress on immune function: Implications for immunoprotection versus immunopathology. Allergy Asthma Clin. Immunol. 4:2-11.

Dhingra, R., L. C. Kwee, D. Diaz-Sanchez et al. 2019. Evaluating DNA methylation age on the Illumina MethylationEPIC Bead Chip. PLoS One 14:1-14.

Eisenlohr-Moul, T. A., A. B. Miller, M. Giletta et al. 2018. HPA axis response and psychosocial stress as interactive predictors of suicidal ideation and behavior in adolescent females: A multilevel diathesis-stress framework. Neuropsychopharmacology 43:2,564-2,571.

Fatemi, M., A. Hermann, H. Gowher et al. 2002. Dnmt3a and Dnmt1 functionally cooperate during de novo methylation of DNA. Eur. J. Biochem. 269:4,981-4,984. 
Felix, J. F., and C. A. M. Cecil. 2019. Population DNA methylation studies in the Developmental Origins of Health and Disease (DOHaD) framework. J. Dev. Orig. Health Dis. 10:306313.

Fiorito, G., S. Polidoro, P.-A. Dugué et al. 2017. Social adversity and epigenetic aging: A multicohort study on socioeconomic differences in peripheral blood DNA methylation. Sci. Rep. 7:1-12.

Fortin, J.-P., A. Labbe, M. Lemire et al. 2014. Functional normalization of 450k methylation array data improves replication in large cancer studies. Genome Biol. 15:1-17.

Garg, P., R. S. Joshi, C. Watson et al. 2018. A survey of inter-individual variation in DNA methylation identifies environmentally responsive co-regulated networks of epigenetic variation in the human genome. PLoS Genet. 14:1-24.

Hashimshony, T., J. Zhang, I. Keshet et al. 2003. The role of DNA methylation in setting up chromatin structure during development. Nat. Genet. 34:187-192.

Hata, K., M. Okano, H. Lei et al. 2002. Dnmt3L cooperates with the Dnmt3 family of de novo DNA methyltransferases to establish maternal imprints in mice. Development 129:1,983$1,993$.

Heijmans, B. T., E. W. Tobi, A. D. Stein et al. 2008. Persistent epigenetic differences associated with prenatal exposure to famine in humans. Proc. Natl. Acad. Sci. U. S. A. 105:17,04617,049 .

Höhne, N., M. Poidinger, F. Merz et al. 2014. Increased HPA axis response to psychosocial stress in remitted depression: The influence of coping style. Biol. Psychol. 103:267-275.

Horvath, S. 2013. DNA methylation age of human tissues and cell types. Genome Biol. 14:1-19. 
Horvath, S., M. Gurven, M. E. Levine et al. 2016. An epigenetic clock analysis of race/ethnicity, sex, and coronary heart disease. Genome Biol. 17:1-22.

Horvath, S., J. Oshima, G. M. Martin et al. 2018. Epigenetic clock for skin and blood cells applied to Hutchinson Gilford Progeria Syndrome and ex vivo studies. Aging (Albany NY) $10: 1,758-1,775$.

Houseman, E. A., W. P. Accomando, D. C. Koestler et al. 2012. DNA methylation arrays as surrogate measures of cell mixture distribution. BMC Bioinformatics 13:1-16.

Hu, Y.-G., R. Hirasawa, J.-L. Hu et al. 2008. Regulation of DNA methylation activity through Dnmt3L promoter methylation by Dnmt3 enzymes in embryonic development. Hum. Mol. Genet. 17:2,654-2,664.

Jaffe, A. E., P. Murakami, H. Lee et al. 2012. Bump hunting to identify differentially methylated regions in epigenetic epidemiology studies. Int. J. Epidemiol. 41:200-209.

Jahanshad, N., P. Rajagopalan, X. Hua et al. 2013. Genome-wide scan of healthy human connectome discovers SPON1 gene variant influencing dementia severity. Proc. Natl. Acad. Sci. U. S. A. 110:4,768-4,773.

Jawaid, A., M. Roszkowski, and I. M. Mansuy. 2018. Transgenerational epigenetics of traumatic stress. In Progress in Molecular Biology and Translational Science: Neuroepigenetics and Mental Illness, vol. 158, B. P. F. Rutten, ed. Cambridge, MA: Academic Press, 273298.

Kertes, D. A., S. S. Bhatt, H. S. Kamin et al. 2017. BNDF methylation in mothers and newborns is associated with maternal exposure to war trauma. Clin. Epigenetics 9:1-12. 
Kertes, D. A., H. S. Kamin, D. A. Hughes et al. 2016. Prenatal maternal stress predicts methylation of genes regulating the hypothalamic-pituitary-adrenocortical system in mothers and newborns in the Democratic Republic of Congo. Child Dev. 87:61-72.

Kim, Y., and T. Maruki. 2011. Hitchhiking effect of a beneficial mutation spreading in a subdivided population. Genetics 189:213-226.

Kraiczy, J., K. M. Nayak, K. J. Howell et al. 2019. DNA methylation defines regional identity of human intestinal epithelial organoids and undergoes dynamic changes during development. Gut 68:49-61.

Lee, J. Y., and T.-H. Lee. 2012. Effects of DNA methylation on the structure of nucleosomes. $J$. Am. Chem. Soc. 134:173-175.

Lehrner, A., and R. Yehuda. 2018. Trauma across generations and paths to adaptation and resilience. Psychol. Trauma 10:22-29.

Lister, R., M. Pelizzola, R. H. Dowen et al. 2009. Human DNA methylomes at base resolution show widespread epigenomic differences. Nature 462:315-322.

Manousaki, D., T. Dudding, S. Haworth et al. 2017. Low-frequency synonymous coding variation in $C Y P 2 R 1$ has large effects on vitamin D levels and risk of multiple sclerosis. Am. J. Hum. Genet. 101:227-238.

Massart, R., M. Freyburger, M. Suderman et al. 2014. The genome-wide landscape of DNA methylation and hydroxymethylation in response to sleep deprivation impacts on synaptic plasticity genes. Transl. Psychiatry 4:1-8.

Mehta, D., T. Klengel, K. N. Conneely et al. 2013. Childhood maltreatment is associated with distinct genomic and epigenetic profiles in posttraumatic stress disorder. Proc. Natl. Acad. Sci. U. S. A. 110:8,302-8,307. 
Min, J. L., G. Hemani, G. Davey Smith et al. 2018. Meffil: Efficient normalization and analysis of very large DNA methylation datasets. Bioinformatics 34:3,983-3,989.

Montoya-Williams, D., J. Quinlan, C. J. Clukay et al. 2018. Associations between maternal prenatal stress, methylation changes in IGF1 and IGF2, and birth weight. J. Dev. Orig. Health Dis. 9:215-222.

Mulligan, C. J. 2016. Early environments, stress, and the epigenetics of human health. Annu. Rev. Anthropol. 45:233-249.

Mulligan, C. J., N. C. D'Errico, J. Stees et al. 2012. Methylation changes at NR3C1 in newborns associate with maternal prenatal stress exposure and newborn birth weight. Epigenetics $7: 853-857$.

Nieratschker, V., R. Massart, M. Gilles et al. 2014. MORC1 exhibits cross-species differential methylation in association with early life stress as well as genome-wide association with MDD. Transl. Psychiatry 4:1-8.

Quinlan, J., L. N. Pearson, C. J. Clukay et al. 2016. Genetic loci and novel discrimination measures associated with blood pressure variation in African Americans living in Tallahassee. PLoS One 11:1-22.

R Development Core Team. 2015. R: A Language and Environment for Statistical Computing. Vienna: R Foundation for Statistical Computing. https://www.R-project.org.

Roadmap Epigenomics Consortium, A. Kundaje, W. Meuleman et al. 2015. Integrative analysis of 111 reference human epigenomes. Nature 518:317-330.

Rodney, N. C., and C. J. Mulligan. 2014. A biocultural study of the effects of maternal stress on mother and newborn health in the Democratic Republic of Congo. Am. J. Phys. Anthropol. 155:200-209. 
Sherva, R., Y. Tripodis, D. A. Bennett et al. 2014. Genome-wide association study of the rate of cognitive decline in Alzheimer's disease. Alzheimers Dement. 10:45-52.

Shields, A. E., L. A. Wise, E. A. Ruiz-Narvaez et al. 2016. Childhood abuse, promoter methylation of leukocyte $N R 3 C 1$ and the potential modifying effect of emotional support. Epigenomics 8:1,507-1,517.

Smith, S. G., X. Zhang, K. C. Basile et al. 2018. The National Intimate Partner and Sexual Violence Survey: 2015 Data Brief-Updated Release. Atlanta, GA: National Center for Injury Prevention and Control, Centers for Disease Control and Prevention. https://www.cdc.gov/violenceprevention/pdf/2015data-brief508.pdf

Smith, Z. D., and A. Meissner. 2013. DNA methylation: Roles in mammalian development. Nat. Rev. Genet. 14:204-220.

Suderman, M., N. Borghol, J. J. Pappas et al. 2014. Childhood abuse is associated with methylation of multiple loci in adult DNA. BMC Med. Genomics 7:1-12.

Sun, B. B., J. C. Maranville, J. E. Peters et al. 2018. Genomic atlas of the human plasma proteome. Nature 558:73-79.

Svedruzić, Z. M. 2008. Mammalian cytosine DNA methyltransferase Dnmt1: Enzymatic mechanism, novel mechanism-based inhibitors, and RNA-directed DNA methylation. Curr. Med. Chem. 15:92-106.

Tobi, E. W., J. J. Goeman, R. Monajemi et al. 2014. DNA methylation signatures link prenatal famine exposure to growth and metabolism. Nat. Commun. 5:1-13.

Verhoeven, J. E., R. Yang, O. M. Wolkowitz et al. 2018. Epigenetic age in male combat-exposed war veterans: Associations with posttraumatic stress disorder status. Mol. Neuropsychiatry 4:90-99. 
Wagner, J. R., S. Busche, B. Ge et al. 2014. The relationship between DNA methylation, genetic and expression inter-individual variation in untransformed human fibroblasts. Genome Biol. 15:1-17.

Youn, A., and S. Wang. 2018. The MiAge Calculator: A DNA methylation-based mitotic age calculator of human tissue types. Epigenetics 13:192-206.

Zannas, A. S., J. Arloth, T. Carrillo-Roa et al. 2015. Lifetime stress accelerates epigenetic aging in an urban, African American cohort: Relevance of glucocorticoid signaling. Genome Biol. 16:1-12.

Zhou, W., P. W. Laird, and H. Shen. 2017. Comprehensive characterization, annotation and innovative use of Infinium DNA methylation BeadChip probes. Nucleic Acids Res. 45:112.

Zovkic, I. B., and J. D. Sweatt. 2013. Epigenetic mechanisms in learned fear: Implications for PTSD. Neuropsychopharmacology 38:77-93. 
Table 1. Summary of Maternal $(n=24)$ and Newborn $(n=22)$ Sample Characteristics

\begin{tabular}{|l|c|}
\hline \multicolumn{1}{|c|}{ Variable } & Mean (s.d.) \\
& [or Count for Categorical Variable] \\
\hline Maternal Variables: & $26.9(5.50)$ \\
\hline Age & $1.58(2.21)$ \\
\hline War Trauma & $6.54(6.32)$ \\
\hline Chronic Stress & $0.625(1.09)$ \\
\hline Sexual Violence & 12 \\
\hline & 10 \\
\hline Newborn Variables: & $7.00(1.69)$ \\
\hline Males & \\
\hline Females & \\
\hline Birthweight (lbs.) & \\
\hline
\end{tabular}


Table 2. Associations between Maternal Stress and Newborn Birthweight Using Simple Regression

\begin{tabular}{|l|c|c|c|}
\hline & \multicolumn{3}{|c|}{ Birthweight (lbs.) } \\
\hline & $\beta$ & $\mathrm{R} 2$ & $\mathrm{p}$-value \\
\hline War Trauma & -0.49 & 0.40 & $9.0 \times 10-4$ \\
\hline Chronic Stress & -0.18 & 0.46 & $3.0 \times 10-4$ \\
\hline Sexual Violence & -0.91 & 0.35 & $2.3 \times 10-3$ \\
\hline
\end{tabular}

Table legend: Effect estimates $(\beta)$, variance explained $\left(\mathrm{R}_{2}\right)$ and $\mathrm{p}$-value estimates for univariate linear models testing the effect of each exposure or stressor on our outcome, birthweight.

Birthweight was not transformed as it was statistically normally with a Shapiro's W-statistic of 0.96 (p-value $=0.42)$. Beta estimates reflect pounds of change per unit stress. 
Table 3. Associations of Summary Methylation Measures with Maternal Stress and

Birthweight, Presented as Beta Values (or Mean Difference between Groups for Crosses Involving Personal Experience of Rape)

\begin{tabular}{|c|c|c|c|c|}
\hline & $\begin{array}{c}\text { War } \\
\text { Trauma }\end{array}$ & $\begin{array}{c}\text { Chronic } \\
\text { Stress }\end{array}$ & $\begin{array}{c}\text { Sexual } \\
\text { Violence }\end{array}$ & Birthweight \\
\hline \multicolumn{5}{|l|}{ Maternal Blood } \\
\hline $\begin{array}{c}\text { GMM, } \\
\text { Beta value }\end{array}$ & $\begin{array}{l}4.0 \times 10-5 \\
(p=0.86)\end{array}$ & $\begin{array}{l}5.7 \times 10-5 \\
(p=0.43)\end{array}$ & $\begin{array}{l}1.2 \times 10-5 \\
(p=0.98)\end{array}$ & $\begin{array}{l}5.2 \times 10-5 \\
(p=0.85)\end{array}$ \\
\hline $\begin{array}{c}\text { Skin \& Blood Clock Age } \\
\text { Acceleration, } \\
\text { Years }\end{array}$ & $\begin{array}{c}0.44 \\
(p=0.27)\end{array}$ & $\begin{array}{c}0.18 \\
(p=0.15)\end{array}$ & $\begin{array}{c}0.64 \\
(p=0.44)\end{array}$ & $\begin{array}{c}-0.32 \\
(p=0.50)\end{array}$ \\
\hline $\begin{array}{l}\text { Mitotic Age, } \\
\text { \# Replications }\end{array}$ & $\begin{array}{c}-4.1 \\
(p=0.56)\end{array}$ & $\begin{array}{c}0.51 \\
(\mathrm{p}=0.82)\end{array}$ & $\begin{array}{c}-12.7 \\
(p=0.38)\end{array}$ & $\begin{array}{c}4.07 \\
(p=0.63)\end{array}$ \\
\hline \multicolumn{5}{|l|}{ Cord Blood } \\
\hline $\begin{array}{c}\text { GMM, } \\
\text { Beta value }\end{array}$ & $\begin{array}{l}-4.8 \times 10-4 \\
(p=0.025)\end{array}$ & $\begin{array}{l}-1.1 \times 10-4 \\
(p=0.14)\end{array}$ & $\begin{array}{l}-7.8 \times 10-4 \\
(p=0.053)\end{array}$ & $\begin{array}{l}5.3 \times 10-4 \\
(\mathrm{p}=0.073)\end{array}$ \\
\hline $\begin{array}{c}\text { Skin \& Blood Clock Age } \\
\text { Acceleration, } \\
\text { Years }\end{array}$ & $\begin{array}{c}5.9 e-03 \\
(p=0.71)\end{array}$ & $\begin{array}{c}2.6 e-03 \\
(p=0.59)\end{array}$ & $\begin{array}{c}0.034 \\
(p=0.20)\end{array}$ & $\begin{array}{c}-0.028 \\
(p=0.16)\end{array}$ \\
\hline $\begin{array}{l}\text { Mitotic Age, } \\
\text { \# Replications }\end{array}$ & $\begin{array}{c}-9.0 \\
(p=0.20)\end{array}$ & $\begin{array}{c}0.54 \\
(p=0.81)\end{array}$ & $\begin{array}{c}-7.9 \\
(p=0.55)\end{array}$ & $\begin{array}{c}9.7 \\
(p=0.31)\end{array}$ \\
\hline
\end{tabular}

Note: Statistically significant association is in bold. 


\section{Figure Captions}

Figure 1. Double-sided Manhattan plot of maternal war trauma (A, B), chronic stress (C, D), sexual violence $(\mathrm{E}, \mathrm{F})$, and newborn birthweight $(\mathrm{G}, \mathrm{H})$ in relation to maternal and cord blood methylation, respectively. Direction of points from zero represents the sign of the beta value for the association. Chromosomes are differentiated by alternating colors and are in numeric order 122 followed by the sex chromosomes. Red lines represent the Bonferroni threshold after multiple testing correction $(0.05 / 412,360 \mathrm{CpG}$ sites $=1.21 \mathrm{e}-7)$.

Figure 2. Partial regression plots illustrating the relationships between the 150 th maternal VMR representative site (cg23284931) and A) maternal chronic stress and B) maternal experience of sexual violence. All graph values were adjusted for cell composition, batch effects, and maternal age. The significance threshold was calculated using Bonferroni correction based on the total number of VMRs identified $(0.05 / 885 \mathrm{CpG}$ sites $=5.65 \mathrm{e}-5)$. The beta and p-values are those determined by the model implemented in meffil for the corresponding association.

Figure 3. Partial regression plot illustrating the relationship of cord blood GMM with maternal war trauma (A) and maternal sexual violence (B). All graph values were adjusted for covariates measuring cell composition, batch effects, and infant sex. Beta, $\mathrm{R}_{2}$, and p-values reflect those found using a multiple regression including the above covariates.

Supplementary Figure S1. Distribution of maternal epigenetic age acceleration values when calculated using A) subtraction of chronological age from epigenetic age B) regression of epigenetic against chronological age, with an intercept value set to zero, and extraction of the residuals. Dashed lines represent the mean value for each figure. 
Figure 1.

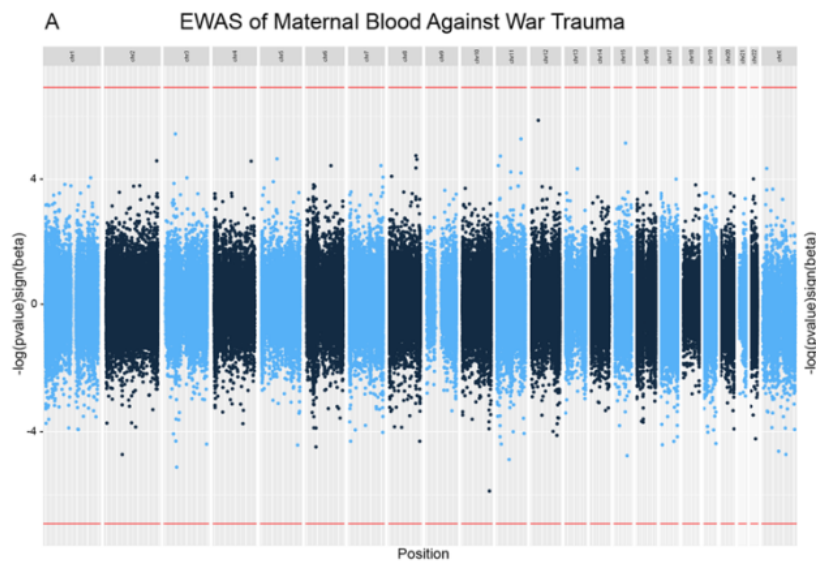

B EWAS of Cord Blood Against War Trauma

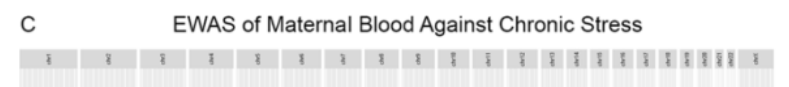

\begin{tabular}{l} 
EWAS of Cord Blood Against Chronic Stress \\
\hline $1+1$ \\
\hline
\end{tabular}
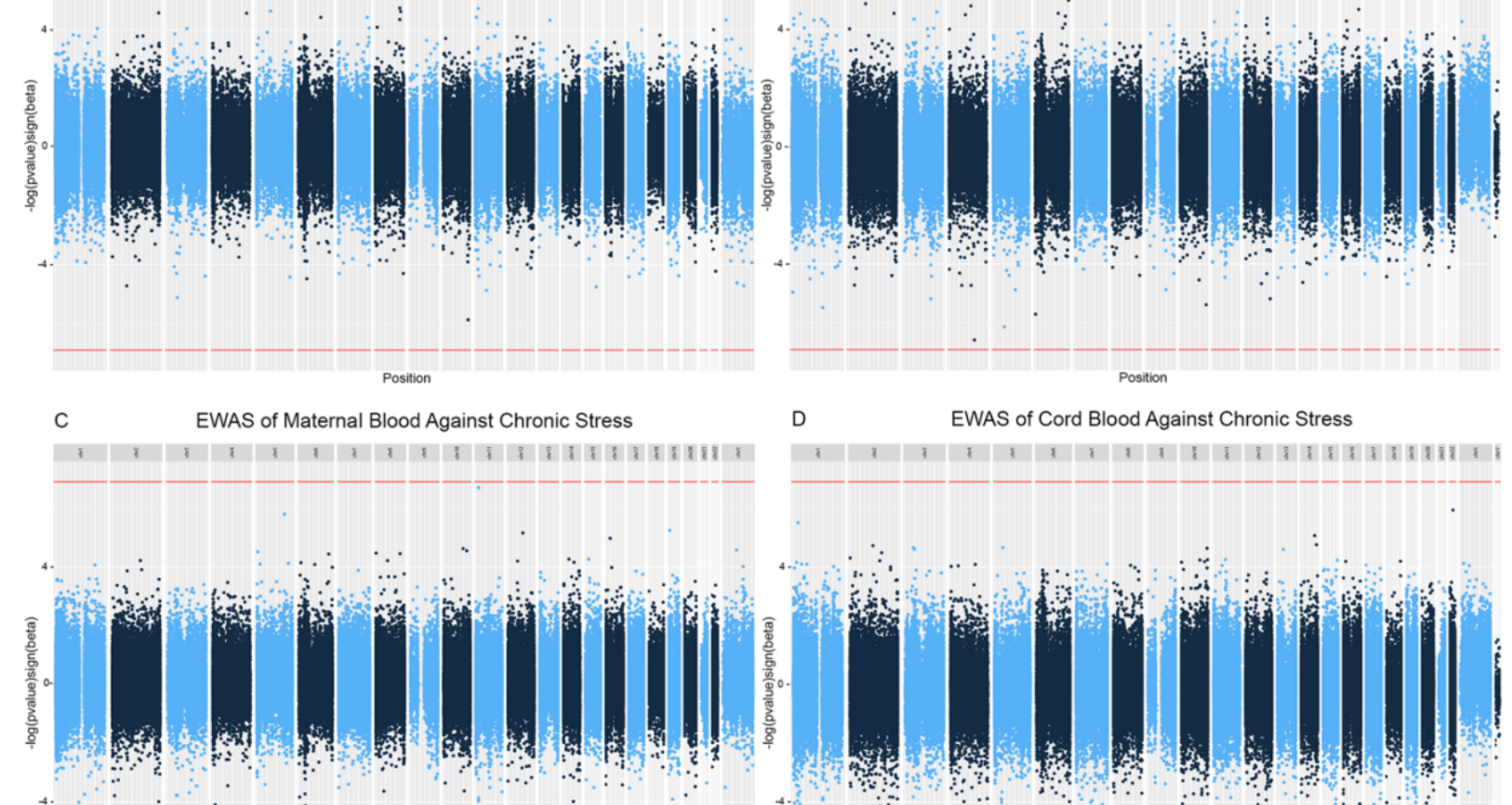

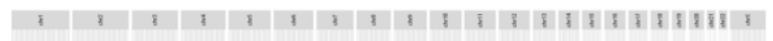
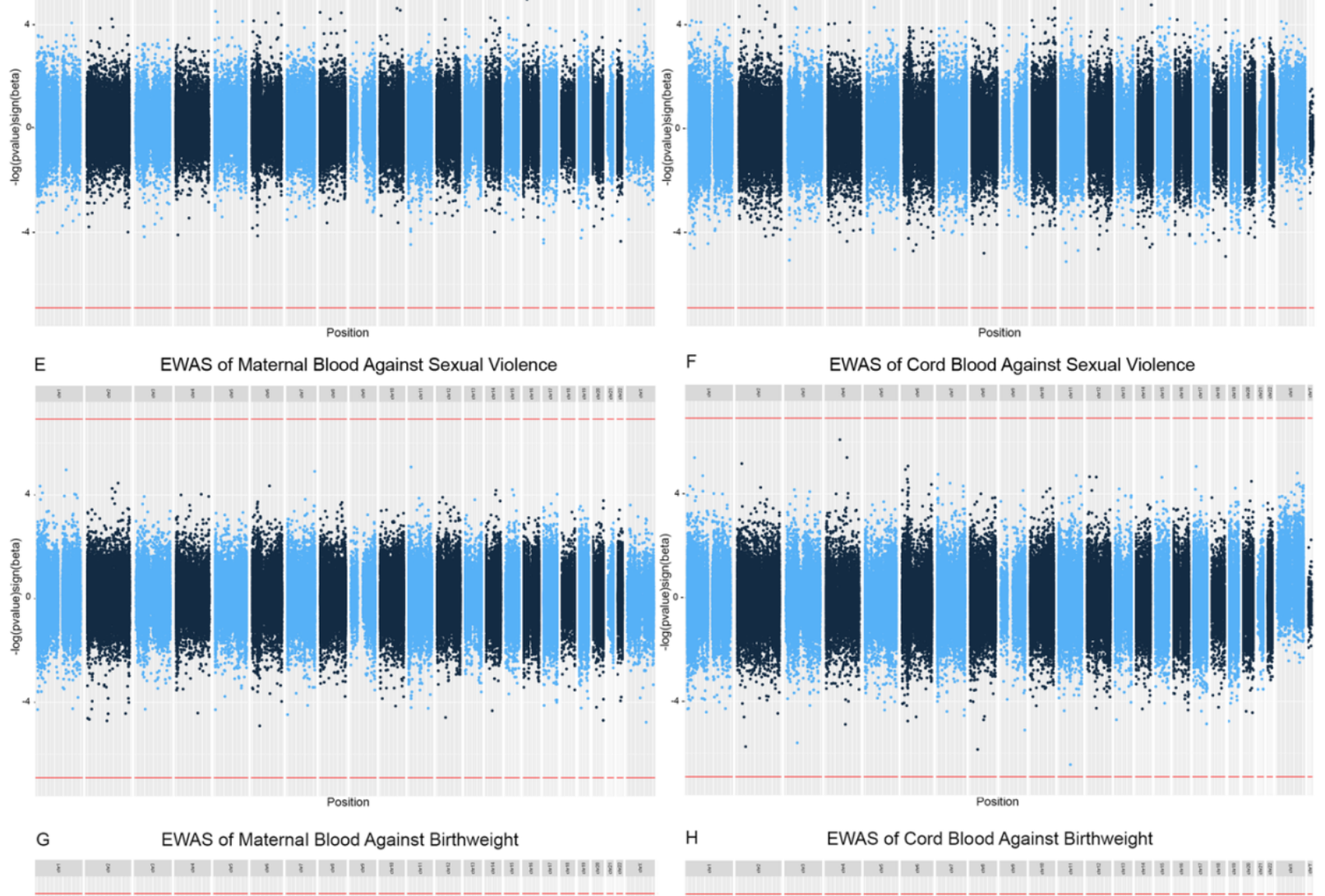

\begin{tabular}{l} 
EWAS of Cord Blood Against Birthweight \\
\hline $1+1$ \\
\hline
\end{tabular}

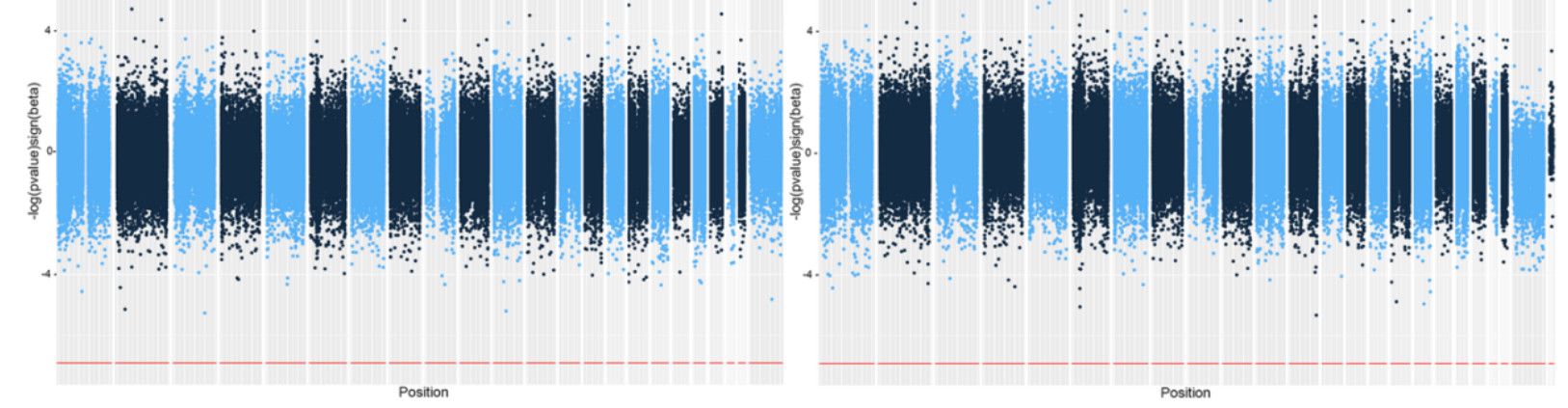

Pre-print version. Visit http://digitalcommons.wayne.edu/humbiol/ after publication to acquire the final version. 
Figure 2.

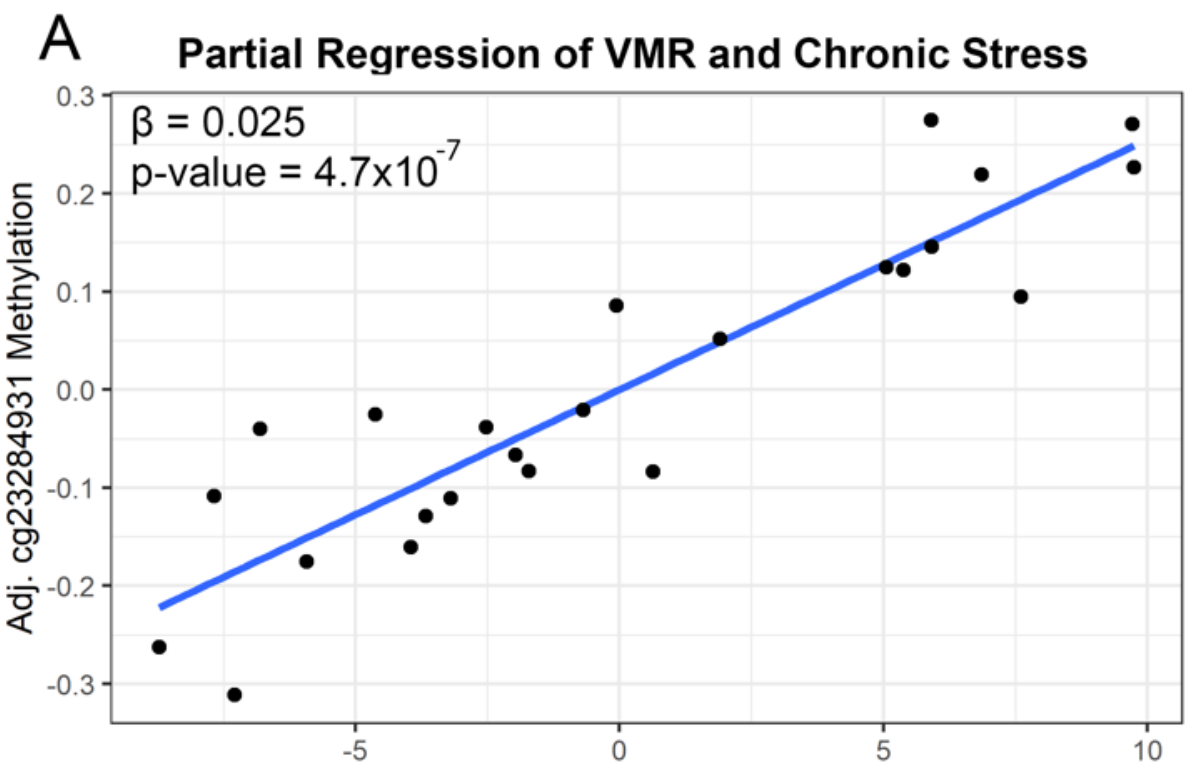

Adj. Chronic Stress

\section{B Partial Regression of VMR and Sexual Violence}

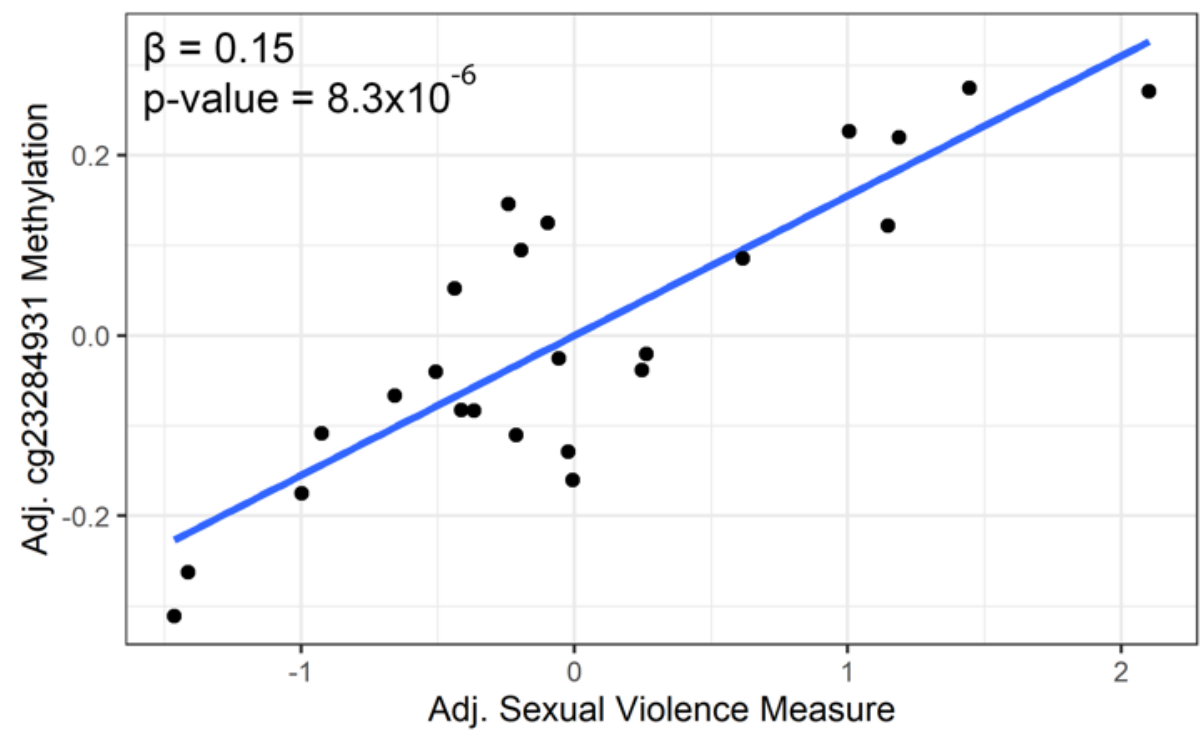

Pre-print version. Visit http://digitalcommons.wayne.edu/humbiol/ after publication to acquire the final version. 
Figure 3.
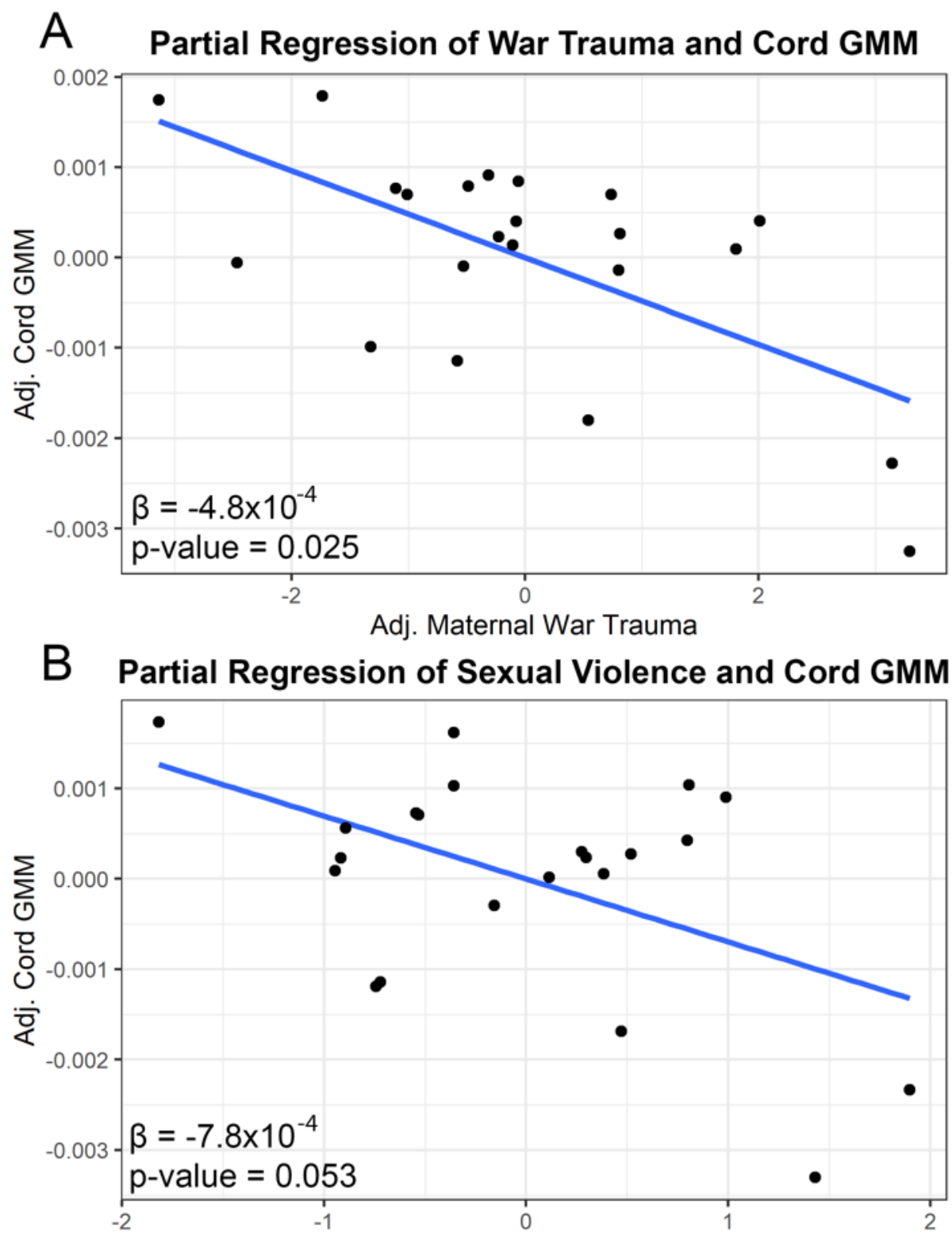

Adj. Sexual Violence Measure 
Supplementary Figure S1.

A

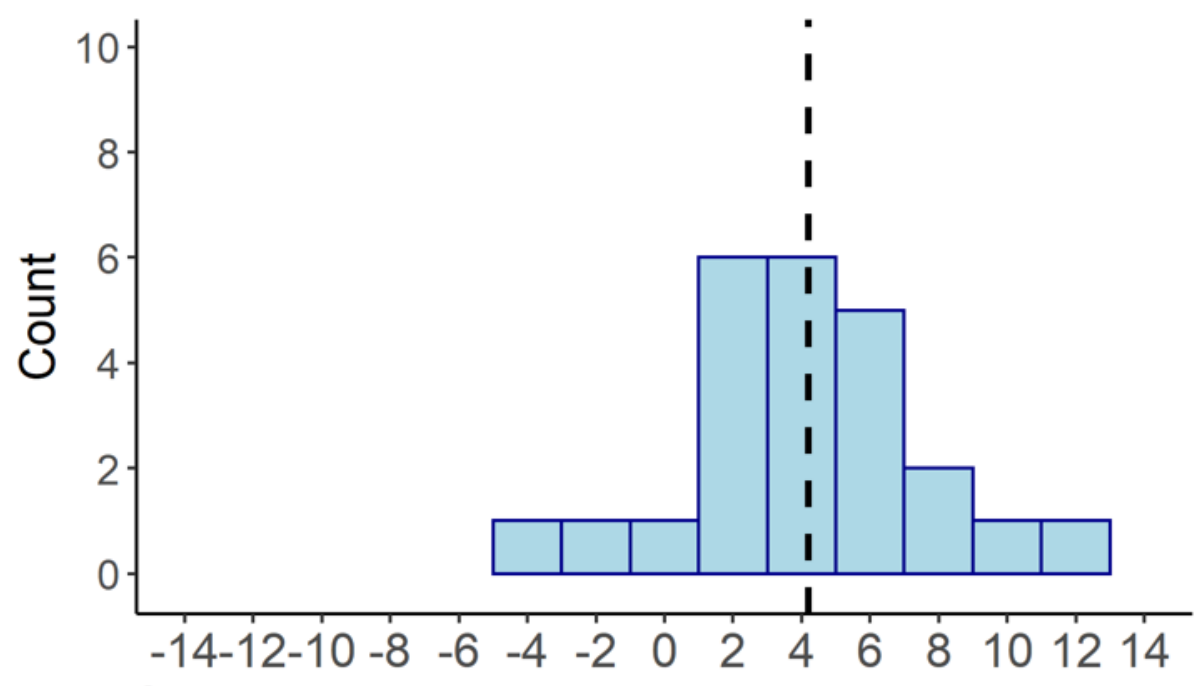

B

Subtraction Adj. Methylation Age Acceleration (Yr)

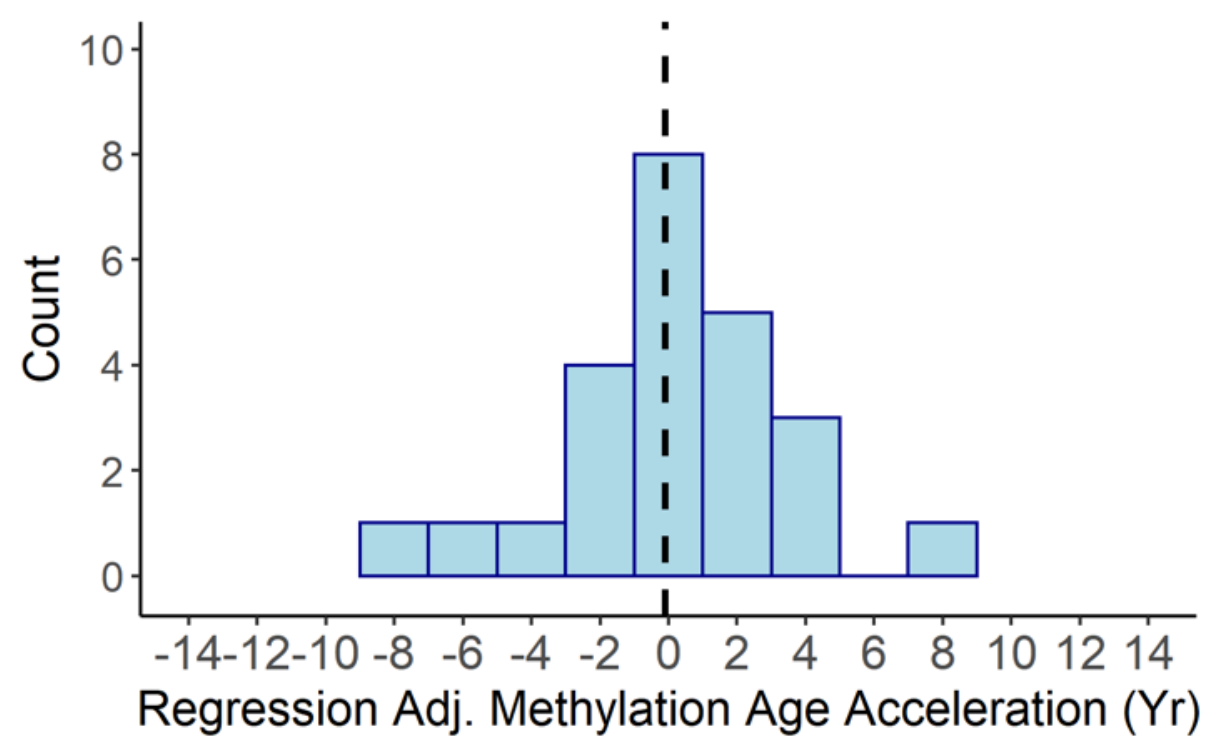

